PROCEEDINGS OF THE AMERICAN MATHEMATICAL SOCIETY

Volume 124, Number 11, November 1996

\title{
GENERIC VANISHING FOR HARMONIC SPINORS OF TWISTED DIRAC OPERATORS
}

\author{
NICOLAE ANGHEL
}

(Communicated by Peter Li)

\begin{abstract}
In this paper we address the problem of generic vanishing for (negative) harmonic spinors of Dirac operators coupled with variable metric connections.
\end{abstract}

\section{IntRoduction}

Let $M$ be a compact spin manifold with spinor bundle $S$ and Dirac operator $\not \partial$. In $[\mathrm{L}]$ Lichnerowicz proved a very strong vanishing theorem: $\not \partial$ has no harmonic spinors ( $\operatorname{dim} \operatorname{ker} \not \partial=0)$, if the scalar curvature of $M$ is non-negative and does not vanish identically (see [LM] for complete details). When $M$ is even-dimensional, $S$ splits into a direct sum of positive and negative spinors, $S=S^{+} \oplus S^{-}$. In this case it would be nice to have a less restrictive Lichnerowicz-type theorem, ensuring the vanishing of, say, only the negative harmonic spinors. To our knowledge, such a theorem does not exist for arbitrary $M$. There is evidence, though, that it should (cf. $[\mathrm{CM}]$, where $M$ is a homogeneous space).

On a related note, in $[\mathrm{S}]$ Singer stressed the importance of vanishing of negative harmonic spinors in particle physics. He claimed, without proof, that for twisted Dirac operators $\not \partial_{A}$, obtained by coupling $(S, \not \partial)$ with a fixed Hermitian vector bundle $E$ equipped with a variable metric connection $A$, this vanishing is generic in $A$. Here, of course, index $\left(\not \partial_{A}^{+}\right)$, which is independent of $A$, is assumed to be non-negative.

In this paper we address the problem of generic vanishing for (negative) harmonic spinors for Dirac operators coupled with variable metric connections. Our treatment is elementary and direct, avoiding the customary use of the Sard-Smale theorem. In fact, we feel that, for the problem at hand, this direct way is better suited and definitely more revealing.

First we develop a general tool for analyzing $\min _{A \in \mathcal{C}} \operatorname{dim} \operatorname{ker}(D+A)$, where $\{D+A\}_{A \in \mathcal{C}}$ is a certain family of unbounded Fredholm operators on an arbitrary Hilbert space (Theorems 1.1, 1.2, and 1.3). Next we use this tool to address the generic vanishing problem stated above, when either $\operatorname{dim} M \leq 4$ (Theorem 1.5) or

Received by the editors May 9, 1995.

1991 Mathematics Subject Classification. Primary 58G25; Secondary 47A53, 53C05.

Key words and phrases. Spin manifold, Dirac operator, metric connection, harmonic spinor, generic vanishing.

Research supported in part by the Texas Advanced Research Program under Grant No. 003594071.

(C)1996 American Mathematical Society 
$M$ has arbitrary dimension but its scalar curvature is non-negative, and the bundle $E$ is trivial (Theorem 1.6). Theorem 1.5 should be true in any dimension, but our limited understanding of spinors prevents us from concluding so.

\section{Statement of Results}

Let $(H,\|\cdot\|)$ be a separable complex Hilbert space and let $D$ be an unbounded essentially self-adjoint Fredholm operator on $H$ [RS]. Let $\mathcal{C} \subset \mathcal{L}(H)$ be a topological linear real subspace of bounded self-adjoint operators which are $D$-compact, i.e., compact when viewed as operators from $\operatorname{dom}(D)$, equipped with the Sobolev norm $\left(\|\cdot\|^{2}+\|D \cdot\|^{2}\right)^{\frac{1}{2}}$, to $H$. Then $D+A, A \in \mathcal{C}$, is also a Fredholm operator with domain $\operatorname{dom}(D)[\mathrm{G}]$.

Fix $A \in \mathcal{C}$ and for $0 \leq t \leq 1$ consider the homotopy of Fredholm operators $D_{t}:=D+t A$ connecting $D$ to $D+A$. Denote by $d_{A}:[0,1] \rightarrow \mathbb{N}$ the function $d_{A}(t):=\operatorname{dim} \operatorname{ker}\left(D_{t}\right)$.

Theorem 1.1. The function $d_{A}$ attains its minimum everywhere on $[0,1]$, except for finitely many points.

Let $d_{\text {min }}=\min _{A \in \mathcal{C}} \operatorname{dim} \operatorname{ker}(D+A)$. What can one say about the subset $\mathcal{C}_{\text {min }}$ of $\mathcal{C}$, consisting of operators $A$ such that $\operatorname{dim} \operatorname{ker}(D+A)=d_{\min }$ ? Theorem 1.1 immediately yields the following answer.

Theorem 1.2. The subset $\mathcal{C}_{\min }$ of $\mathcal{C}$ is open and dense in $\mathcal{C}$.

Theorem 1.2, in turn, yields a useful necessary and sufficient condition guaranteeing that $\operatorname{dim} \operatorname{ker}(D)=d_{\text {min }}$, i.e., $0 \in \mathcal{C}_{\text {min }}$. In order to state it we need more preparation.

The Fredholmness of $D$ implies that in the orthogonal splitting $H=(\operatorname{ker} D) \oplus$ $(\operatorname{ker} D)^{\perp}, \operatorname{ker} D$ is finite dimensional and $D$ can be represented as

$$
D=\left[\begin{array}{ll}
0 & 0 \\
0 & \delta
\end{array}\right]
$$

where $\delta:(\operatorname{ker} D)^{\perp} \cap \operatorname{dom}(D) \rightarrow(\operatorname{ker} D)^{\perp}$ is a linear isomorphism such that, for some $k>0$,

$$
\|\delta(x)\| \geq k\|x\|, \quad \text { for any } x \in(\operatorname{ker} D)^{\perp} \cap \operatorname{dom}(D) .
$$

With respect to the same splitting we have, for $A \in \mathcal{C}$,

$$
D+A=\left[\begin{array}{cc}
a_{A} & b_{A} \\
b_{A}^{*} & \delta+c_{A}
\end{array}\right],
$$

where $a_{A}: \operatorname{ker} D \rightarrow \operatorname{ker} D, c_{A}:(\operatorname{ker} D)^{\perp} \rightarrow(\operatorname{ker} D)^{\perp}$ are bounded self-adjoint linear operators, and $b_{A}:(\operatorname{ker} D)^{\perp} \rightarrow \operatorname{ker} D$ is bounded linear $(a, b, c$ are induced by $A$ ).

Theorem 1.3. In the above notation, $\operatorname{dim} \operatorname{ker} D=d_{\min }$ if and only if

$$
a_{A}=0, b_{A}\left(\delta^{-1} c_{A}\right)^{i} \delta^{-1} b_{A}^{*}=0, i=0,1,2, \ldots, \text { for any } A \in \mathcal{C} .
$$

The above findings will be used to prove generic vanishing for harmonic spinors of Dirac operators on spin manifolds, coupled with variable metric connections. We work only with spin manifolds for convenience. Of course, similar results can be stated for the more general $\operatorname{spin}^{c}$-manifolds. To establish the notation and for the 
convenience of the reader we recall here the main concepts regarding twisted Dirac operators (cf. [GL], [LM] for details).

Let $M$ be a compact oriented Riemannian spin manifold of dimension $n$ and let $S$ be the complex bundle of spinors over $M$ with canonical Riemannian connection $\nabla$. $S$ has rank $2^{\left[\frac{n}{2}\right]}$. When $n$ is even, Clifford multiplication by the volume form of $M$ gives rise to a self-adjoint involution $\tau$ on $S$. With respect to this involution $S$ splits into a direct sum of positive and negative spinors $S=S^{+} \oplus S^{-}, S^{ \pm}:=\{\tau= \pm 1\}$. Let $\not \partial$ be the Dirac operator on $\mathcal{C}^{\infty}(S)$, associated to the Clifford multiplication on $S$ by tangent vectors to $M$ and the connection $\nabla$. $\not \partial$ is a first order elliptic differential operator, which locally, with respect to an orthonormal basis $\left\{e_{i}\right\}_{i=1}^{n}$ of $M$, is expressible as

$$
\not \partial=\sum_{i=1}^{n} e_{i} \cdot \nabla_{e_{i}}
$$

The unique closure of $\not \partial$ to the $L^{2}$-space $L^{2}(S)$, still denoted by $\not \partial$, makes $\not \partial$ an essentially self-adjoint Fredholm operator. When $n$ is even, $\not \partial$ anticommutes with $\tau$, giving rise to half-Dirac operators $\not^{ \pm}: \mathcal{C}^{\infty}\left(S^{ \pm}\right) \rightarrow \mathcal{C}^{\infty}\left(S^{\mp}\right)$.

Now fix a Hermitian vector bundle $E$ over $M$. Denote by $\mathcal{A}$ the affine space of $\mathcal{C}^{\infty}$-Hermitian (metric) connections on $E$, equipped with the $\mathcal{C}^{\infty}$-topology. For $A \in \mathcal{A}$ let $\nexists_{A}$ be the twisted Dirac operator on $\mathcal{C}^{\infty}(S \otimes E)$, associated, via Equation (1.4) to the tensor product connection $\nabla \otimes I d+I d \otimes A$ on $S \otimes E$. Again, $\not_{A}$ is an essentially self-adjoint Fredholm operator on $L^{2}(S \otimes E)$. $\not_{A}^{ \pm}$will denote the restriction of $\partial_{A}$ to $\mathcal{C}^{\infty}\left(S^{ \pm} \otimes E\right)$, if $M$ is even-dimensional.

Finally, index $\left(\not_{A}\right)=0$, since $\not_{A}$ is a self-adjoint operator. If $A_{1}, A_{2} \in \mathcal{A}$, $\not \partial_{A_{1}}$ differs from $\not \partial_{A_{2}}$ by a $0^{\text {th }}$-order term. Thus index $\left(\not_{A_{1}}^{+}\right)=\operatorname{index}\left(\not_{A_{2}}^{+}\right)$, in the even-dimensional case. The Atiyah-Singer index theorem [AS] equates precisely index $\left(\not \partial_{A}^{+}\right)$with $\int_{M} \operatorname{ch}(E) \cdot \widehat{A}(M)$, where $\operatorname{ch}(E)$ is the Chern character of $E$ and $\widehat{A}(M)$ is the total $\widehat{A}$-class of $M$. We will assume that $\int_{M} \operatorname{ch}(E) \cdot \widehat{A}(M) \geq 0$.

A section $s \in \mathcal{C}^{\infty}(S \otimes E)$ such that $\not_{A} s=0$ will be called a harmonic spinor for the twisted Dirac operator $\not_{A}$. Likewise, $s \in \mathcal{C}^{\infty}\left(S^{-} \otimes E\right), \not \partial_{A}^{-} s=0$, will be called a negative harmonic spinor. (Negative) Harmonic spinors tend to vanish generically in $A$, as the following results state.

Theorem 1.5. For an open and dense set of metric connections $A \in \mathcal{A}$, the twisted Dirac operator $\not_{A}$ has no harmonic spinors ( $\left.\operatorname{dim} \operatorname{ker} \not_{A}=0\right)$, respectively negative harmonic spinors $\left(\operatorname{dim} \operatorname{ker} \not \not_{A}^{-}=0\right)$ if $\operatorname{dim} M=1$ or 3 , respectively $\operatorname{dim} M=2$ or 4.

Theorem 1.6. If the compact spin manifold $M$ has arbitrary dimension but its scalar curvature is non-negative and does not vanish identically, and the bundle $E$ is trivial, then the twisted Dirac operator $\not_{A}$ has no harmonic spinors (dim ker $\not_{A}=0$ ) for an open and dense set of metric connections $A \in \mathcal{A}$.

\section{Proofs}

This section contains the proofs of the theorems stated in the previous section. We start with a key lemma.

Lemma 2.1. The function $d: \mathcal{C} \rightarrow \mathbb{N}, d(A):=\operatorname{dim} \operatorname{ker}(D+A)$ is upper semicontinuous on $\mathcal{C}$. 
Proof. Since $d$ is integer-valued, Lemma 2.1 is equivalent to the following statement: for any $A_{0} \in \mathcal{C}$ there is an $\epsilon>0$ such that if $A \in \mathcal{C},\|A\|<\epsilon$, then $d\left(A_{0}+A\right) \leq$ $d\left(A_{0}\right)$. Replacing eventually $D$ by $D+A_{0}$, it is enough to prove this statement when $A_{0}=0$.

To this end, we consider the orthogonal splitting $H=(\operatorname{ker} D) \oplus(\operatorname{ker} D)^{\perp}$, where ker $D$ is finite dimensional. As in Section 1, $D$ will be represented by

$$
D=\left[\begin{array}{ll}
0 & 0 \\
0 & \delta
\end{array}\right]
$$

where $\delta:(\operatorname{ker} D)^{\perp} \cap \operatorname{dom}(D) \rightarrow(\operatorname{ker} D)^{\perp}$ is a linear isomorphism such that, for some $k>0$ (depending on $D)$,

$$
\|\delta(x)\| \geq k\|x\|, \quad \text { for any } x \in(\operatorname{ker} D)^{\perp} \cap \operatorname{dom}(D) .
$$

With respect to the same splitting we have,

$$
D+A=\left[\begin{array}{cc}
a_{A} & b_{A} \\
b_{A}^{*} & \delta+c_{A}
\end{array}\right]
$$

where $a_{A}: \operatorname{ker} D \rightarrow \operatorname{ker} D, c_{A}:(\operatorname{ker} D)^{\perp} \rightarrow(\operatorname{ker} D)^{\perp}$ are bounded self-adjoint linear operators, and $b_{A}:(\operatorname{ker} D)^{\perp} \rightarrow \operatorname{ker} D$ is bounded linear $(a, b, c$ are induced by $A$ ).

Notice that $\left\|c_{A}\right\|<k$, if $A \in \mathcal{C},\|A\|<k$. Then, Equation (2.2) implies that $\delta+c_{A}$ is invertible and we have

$$
\left(\delta+c_{A}\right)^{-1}=\sum_{i=0}^{\infty}(-1)^{i}\left(\delta^{-1} c_{A}\right)^{i} \delta^{-1}
$$

For $\|A\|<k$, the matrix

$$
\left[\begin{array}{cc}
I d & -b_{A}\left(\delta+c_{A}\right)^{-1} \\
0 & \left(\delta+c_{A}\right)^{-1}
\end{array}\right]
$$

clearly induces a one-to-one bounded linear operator on $H$. Since

$$
\left[\begin{array}{cc}
I d & -b\left(\delta+c_{A}\right)^{-1} \\
0 & \left(\delta+c_{A}\right)^{-1}
\end{array}\right](D+A)=\left[\begin{array}{cc}
a_{A}-b_{A}\left(\delta+c_{A}\right)^{-1} b_{A}^{*} & 0 \\
\left(\delta+c_{A}\right)^{-1} b_{A}^{*} & I d
\end{array}\right]
$$

we conclude that

$$
\operatorname{ker}(D+A) \cong\left\{x \in \operatorname{ker}(D) \mid a_{A}(x)-b_{A}\left(\delta+c_{A}\right)^{-1} b_{A}^{*}(x)=0\right\} \subseteq \operatorname{ker}(D),
$$

if $\|A\|<k$. Equivalently, $d(A) \leq d(0)$, if $\|A\|<k$. The lemma follows if we set $\epsilon=k$.

Proof of Theorem 1.1. Denote by $d_{A, \min }$ the minimum value of $d_{A}$ on $[0,1]$. Put $I:=\left\{t \in[0,1] \mid t\right.$ possesses an open neighborhood $I_{t}$ in $[0,1]$ such that $d_{A}(\tau)=$ $d_{A, \min }$, for any $\left.\tau \in I_{t} \backslash\{t\}\right\}$. Since $[0,1]$ is compact, Theorem 1.1 will follow if we prove that $I=[0,1]$.

The set $I$ is clearly non-empty and open in $[0,1]$, since by Lemma 2.1 , applied to the one-dimensional subspace of bounded operators $\mathbb{R} A, d_{A}$ is upper semicontinuous. Showing that $I$ is also closed in $[0,1]$ will conclude the proof.

To this end, let $\left\{t_{k}\right\}_{k=1}^{\infty} \subset I$ be such that $\lim _{k \rightarrow \infty} t_{k}=t$. Obviously $t \in I$ if $d_{A}(t)=d_{A, \min }$. Assume that $d_{A}(t)>d_{A, \min }$. We will apply next the formalism developed in the proof of Lemma 2.1, making in the process the substitutions $D \rightarrow D_{t}, A \rightarrow h A, h \in \mathbb{R}$. Equation (2.3), adapted to this situation, implies then that the map $h \longmapsto a_{A, t}-h b_{A, t}\left(\delta_{t}+h c_{A, t}\right)^{-1} b_{A, t}^{*}$ is analytic in $h$, as a map from 
a certain neighborhood $\{|h|<\epsilon\}$ of 0 in $\mathbb{R}$ to self-adjoint operators on the finite dimensional vector space $\operatorname{ker} D_{t}\left(\epsilon\right.$ can be chosen such that $\left.\epsilon\|A\|<k_{t}\right)$. As a result, in matrix representation with respect to some orthonormal basis of ker $D_{t}$, all the minors of $a_{A, t}-h b_{A, t}\left(\delta_{t}+h c_{A, t}\right)^{-1} b_{A, t}^{*}$ are analytic functions in $h$ near 0 .

Equation (2.4) shows now that on $\{|h|<\epsilon\}$,

$$
\operatorname{rk}\left(a_{A, t}-h b_{A, t}\left(\delta_{t}+h c_{A, t}\right)^{-1} b_{A, t}^{*}\right) \leq d_{A}(t)-d_{A, \min },
$$

and in fact the assumption $\left\{t_{k}\right\}_{k=1}^{\infty} \subset I$ guarantees that there are values of $h$ arbitrarily close to 0 such that $\operatorname{rk}\left(a_{A, t}-h b_{A, t}\left(\delta_{t}+h c_{A, t}\right)^{-1} b_{A, t}^{*}\right)=d_{A}(t)-d_{A, \min }$. Thus all the minors of $a_{A, t}-h b_{A, t}\left(\delta_{t}+h c_{A, t}\right)^{-1} b_{A, t}^{*},|h|<\epsilon$, of order $>d_{A}(t)-$ $d_{A, \text { min }}$ are zero, and there is a minor of order $d_{A}(t)-d_{A \text {,min }}$ which does not vanish identically in any neighborhood of 0 . Being an analytic function in $h$, this minor can have $h=0$ only as an isolated zero. In conclusion,

$$
\operatorname{rk}\left(a_{A, t}-h b_{A, t}\left(\delta_{t}+h c_{A, t}\right)^{-1} b_{A, t}^{*}\right)=d_{A}(t)-d_{A, \min },
$$

for $h$ near $0, h \neq 0$, and this, in conjunction with Equation (2.4), proves that $t \in I$.

Proof of Theorem 1.2. $\mathcal{C}_{\min }$ is obviously an open subset of $\mathcal{C}$, by Lemma 2.1. Fix $A_{0} \in \mathcal{C}_{\text {min }}$. Thus $\operatorname{dim} \operatorname{ker}\left(D+A_{0}\right)=d_{\text {min }}$.

We will prove the density of $\mathcal{C}_{\min }$ in $\mathcal{C}$ by showing that if $A \in \mathcal{C}$, then $t A+$ $(1-t) A_{0} \in \mathcal{C}_{\min }$, for values of $t<1$ arbitrarily close to 1 . This follows by applying Theorem 1.1 to the homotopy of Fredholm operators $\widetilde{D}_{t}=\widetilde{D}+t \widetilde{A}, 0 \leq t \leq 1$, where $\widetilde{D}=D+A_{0}$, and $\widetilde{A}=A-A_{0}$ (clearly Theorem 1.1 holds if $D$ is replaced by $\widetilde{D})$. Specifically, $\min _{0 \leq t \leq 1} \operatorname{dim} \operatorname{ker}\left(\widetilde{D}_{t}\right)=\operatorname{dim} \operatorname{ker}\left(\widetilde{D}_{0}\right)=d_{\min }$ and $\widetilde{D}_{t}=D+$ $\left(t A+(1-t) A_{0}\right)$; therefore $\operatorname{dim} \operatorname{ker}\left(D+\left(t A+(1-t) A_{0}\right)\right)=d_{\min }$, for $t$ close to 1 , by Theorem 1.1.

Proof of Theorem 1.3. Assume that $\operatorname{dim} \operatorname{ker}(D)=d_{\min }$. Then Equation (2.4) implies that $a_{A}-h b_{A}\left(\delta+h c_{A}\right)^{-1} b_{A}^{*}=0$, for any $A \in \mathcal{C}$ and any $h \in \mathbb{R},|h||| A||<k$, where $k$ is given by Equation (2.2). By Equation (2.3), the map

$$
h \longmapsto a_{A}-h b_{A}\left(\delta+h c_{A}\right)^{-1} b_{A}^{*}
$$

is analytic in the neighborhood $\{h \in \mathbb{R}|| h|||A| \mid<k\}$ of 0 in $\mathbb{R}$, and its power series expansion about $h=0$ is

$$
a_{A}-\sum_{i=0}^{\infty}(-1)^{i} h^{i+1} b_{A}\left(\delta^{-1} c_{A}\right)^{i} \delta^{-1} b_{A}^{*} .
$$

Since this map vanishes identically, all the coefficients in the above power series expansion must vanish, i.e., $a_{A}=0, b_{A}\left(\delta^{-1} c_{A}\right)^{i} \delta^{-1} b_{A}^{*}=0, i=0,1,2, \ldots$

Conversely, assume that $a_{A}=0, b_{A}\left(\delta^{-1} c_{A}\right)^{i} \delta^{-1} b_{A}^{*}=0, i=0,1,2, \ldots$, for any $A \in \mathcal{C}$. Using again Equations (2.3) and (2.4), we conclude that $\operatorname{dim} \operatorname{ker}(D+A)=$ $\operatorname{dim} \operatorname{ker}(D)$, throughout the neighborhood $\{\|A\|<k\}$ of 0 in $\mathcal{C}$. By Theorem 1.2, there is some operator $A_{0} \in\{A \in \mathcal{C} \mid\|A\|<k\}$ such that $\operatorname{dim} \operatorname{ker}\left(D+A_{0}\right)=d_{\min }$, and so $\operatorname{dim} \operatorname{ker}(D)=d_{\text {min }}$.

Next we are going to apply the above proven theorems to Dirac operators coupled with variable metric connections (cf. Section 1). Throughout the rest of the paper $H$ will be $L^{2}(S \otimes E), D$ will be a (conveniently chosen) twisted Dirac operator $\not_{A}$, and $\mathcal{C}$ will be manufactured out of $\mathcal{A}$ in the following way: Given any 
connection $A \in \mathcal{A}$, then $\mathcal{A}=\{A\}+\Omega^{1}\left(\operatorname{End}_{s k}(E)\right)$, where $\Omega^{1}\left(\operatorname{End}_{s k}(E)\right)$ denotes the $\mathcal{C}^{\infty}(M)$-module of smooth 1-forms with coefficients in the bundle $\operatorname{End}_{s k}(E)$ of skew-Hermitian endomorphisms of $E . \Omega^{1}\left(\operatorname{End}_{s k}(E)\right)$ acts on $L^{2}(S \otimes E)$ by $0^{\text {th }}$ order differential operators (bundle morphisms). Namely, if $X \in \Omega^{1}\left(\operatorname{End}_{s k}(E)\right)$, $s \otimes v \in(S \otimes E)_{m}, m \in M$, and $\left\{e_{i}\right\}_{i=1}^{m}$ is an orthonormal basis for $T_{m} M$, then

$$
X \cdot(s \otimes v)=\sum_{i=1}^{n}\left(e_{i} \cdot s\right) \otimes X_{e_{i}}(v) .
$$

Then $\mathcal{C}$ will denote the topological real subspace of bounded self-adjoint operators on $L^{2}(S \otimes E)$ induced by the continuous action of $\Omega^{1}\left(\operatorname{End}_{s k}(E)\right)$ on $L^{2}(S \otimes E)$. Notice that $\mathcal{C}$ is set up such that

$$
\not \partial_{A+X}=\not \partial_{A}+X \cdot, \quad A \in \mathcal{A}, X \in \Omega^{1}\left(\operatorname{End}_{s k}(E)\right) .
$$

In addition, $\left.\mathcal{C} \cdot L^{2}\left(S^{ \pm} \otimes E\right)\right) \subset L^{2}\left(S^{\mp} \otimes E\right)$, in the even-dimensional case.

Proof of Theorem 1.5. Assume first that $\operatorname{dim} M=n$ is odd. Choose $A_{0} \in \mathcal{A}$ such that $\operatorname{dim} \operatorname{ker}\left(\not \supset_{A_{0}}\right)=d_{\min }$. By Theorem 1.2, it is enough to prove that $d_{\min }=0$, i.e., $\operatorname{dim} \operatorname{ker}\left(\not \partial_{A_{0}}\right)=0$.

Let $u \in \operatorname{ker}\left(\not_{A_{0}}\right)$. By standard regularity arguments, $u \in \mathcal{C}^{\infty}(S \otimes E)$. Using now Theorem 1.3, applied to $D=\not \partial_{A_{0}}$, we conclude that $a_{X}(u)=0$, for any $X \in$ $\Omega^{1}\left(\operatorname{End}_{s k}(E)\right)$. In particular, $\left(a_{X}(u), u\right)=0$, where $(\cdot, \cdot)$ denotes the global inner product on $L^{2}(S \otimes E)$. Equivalently, $(X \cdot u, u)=0$. Since $\Omega^{1}\left(\operatorname{End}_{s k}(E)\right)$ is a $\mathcal{C}^{\infty}(M)$-module, $(X \cdot u, u)=0$ is further equivalent to $\langle X \cdot u, u\rangle=0$, where $\langle\cdot, \cdot \cdot\rangle$ stands for the pointwise inner product on $S \otimes E$.

Assume that $u \not \equiv 0$. Then there is an open subset $U \subset M$ over which all the bundles involved are trivial and such that $\left.u\right|_{U}$ does not vanish. If $\left\{v_{\alpha}\right\}_{\alpha=1}^{p}$, $p=\operatorname{rank}(E)$, is a local orthonormal basis of $E$ over $U$, then

$$
u=\sum_{\alpha=1}^{p} s_{\alpha} \otimes v_{\alpha}, \quad s_{\alpha} \in \mathcal{C}^{\infty}(U, S) .
$$

By Equation (2.5),

$$
\langle X \cdot u, u\rangle=\left\langle\sum_{i, \alpha} e_{i} \cdot s_{\alpha} \otimes X_{e_{i}}\left(v_{\alpha}\right), \sum_{\beta} s_{\beta} \otimes v_{\beta}\right\rangle=\sum_{i, \alpha, \beta}\left\langle e_{i} \cdot s_{\alpha}, s_{\beta}\right\rangle\left\langle X_{e_{i}}\left(v_{\alpha}\right), v_{\beta}\right\rangle .
$$

Since $X_{e_{i}} \in \mathcal{C}^{\infty}\left(U, \operatorname{End}_{s k}\left(\left.E\right|_{U}\right)\right)$ varies arbitrarily, we see that the equation $\langle X \cdot u, u\rangle=0$ is equivalent to

$$
\left\langle e_{i} \cdot s_{\alpha}, s_{\beta}\right\rangle=0, \text { on } U, \text { for any } i, \alpha, \beta .
$$

Shrinking $U$ if necessary, we may assume that for some particular index $\alpha, s_{\alpha}$ does not vanish on $U$. Basic properties of Clifford multiplication show that $\left\{e_{i} \cdot s_{\alpha}\right\}_{i=1}^{n}$ is an $\mathbb{R}$-linearly independent set in $\left.S\right|_{U}$. Since $n$ is odd, the complex dimension of $S$ is $2^{\frac{n-1}{2}}$. As a result, $\left\{e_{i} \cdot s_{\alpha}\right\}_{i=1}^{n}$ generate $\left.S\right|_{U}$ over $\mathbb{C}$, if $n=1$ or 3 . Indeed, the case $n=1$ is obvious ( $\operatorname{rank} S=1$ ), and if $n=3$ the claim is equivalent to the simple fact that three $\mathbb{R}$-linearly independent vectors generate (over $\mathbb{C}$ ) a complex vector space of complex dimension 2. Equation (2.6) now implies that $s_{\alpha} \equiv 0$, a contradiction. Thus $u=0$, i.e., $\operatorname{ker}\left(\not_{A_{0}}\right)=0$. If $\operatorname{dim} m \geq 5$, it is not possible to conclude that $u=0$ just from $a_{X}(u)=0$. However, we do expect Theorem 1.5 to continue to hold, based on the fact that in the arguments above we have not used 
the full strength of Theorem 1.3. Unfortunately, we do not have a proof for this yet.

Assume now that $\operatorname{dim} M=n$ is even and $\int_{M} \operatorname{ch}(E) \cdot \widehat{A}(M) \geq 0$. We must show that if $n=2$ or 4 , then $\operatorname{dim} \operatorname{ker}\left(\not_{A}^{-}\right)=0$, for an open and dense set of metric connections $A$ on $E$. The proof will only be sketched, since it is similar to the one given in odd dimensions.

As before, choose $A_{0} \in \mathcal{A}$ such that $\operatorname{dim} \operatorname{ker}\left(\partial_{A_{0}}\right)=d_{\text {min }}$. The claim is obvious if $d_{\text {min }}=0$, via Theorem 1.2 , so we assume that $d_{\text {min }}>0$. This assumption guarantees the existence of a non-trivial positive harmonic spinor $u^{+} \in \operatorname{ker}\left(\not \partial_{A_{0}}^{+}\right)$. $u^{+}$does not vanish on some coordinate patch $U \subset M$, and if $\left\{v_{\alpha}\right\}_{\alpha=1}^{p}$ is a local orthonormal basis for $\left.E\right|_{U}$, then $\left.u^{+}\right|_{U}=\sum_{\alpha=1}^{p} s_{\alpha} \otimes v_{\alpha}, s_{\alpha} \in \mathcal{C}^{\infty}\left(U, S^{+}\right)$. Thus $\left\{e_{i} \cdot s_{\alpha}\right\}_{i=1}^{n}$ is an $\mathbb{R}$-linearly independent subset of $\left.S^{-}\right|_{U}$, for some index $\alpha$ for which $s_{\alpha}$ does not vanish on $U$. Since the $\mathbb{R}$-rank of $S^{-}$is $2^{\frac{n}{2}}$, we conclude that $\left\{e_{i} \cdot s_{\alpha}\right\}_{i=1}^{n}$ is an $\mathbb{R}$-basis of $S^{-} \mid U$ if $n=2^{\frac{n}{2}}$, i.e., $n=2$ or 4 .

Now let $u^{-} \in \operatorname{ker}\left(\not_{A_{0}}^{-}\right)$. By Theorem 1.3, $a_{X}\left(u^{+}\right)=0$, for any form $X \in$ $\Omega^{1}\left(\operatorname{End}_{s k}(E)\right)$. In particular, $\left(X \cdot u^{+}, u^{-}\right)=0$, or equivalently $\left\langle X \cdot u^{+}, u^{-}\right\rangle=0$. If $\left.u^{-}\right|_{U}=\sum_{\beta=1}^{p} \sigma_{\beta} \otimes v_{\beta}, \sigma_{\beta} \in \mathcal{C}^{\infty}\left(U, S^{-}\right)$, we conclude again that $\left\langle e_{i} \cdot s_{\alpha}, \sigma_{\beta}\right\rangle=0$, for any $i, \alpha, \beta$. Thus $\left.u^{-}\right|_{U} \equiv 0$, if $n=2$ or 4 . Using the principle of analytic continuation for solutions of Dirac equations $[\mathrm{BW}]$, we have that $u^{-}=0$, i.e., $\operatorname{ker}\left(\partial_{A_{0}}^{-}\right)=0$. Yet another use of Theorem 1.2 concludes the proof.

Proof of Theorem 1.6. If the bundle $E$ is trivial and $A_{\text {trivial }}$ is the trivial connection

of $E$ (with respect to some fixed trivialization), then $\not_{A_{\text {trivial }}} \cong \oplus^{\text {rankE}} \not \partial$. Thus $\operatorname{ker}\left(\not_{A_{\text {trivial }}}\right)=0$, by Lichnerowicz's theorem [L]. The theorem follows now from Theorem 1.2.

\section{REFERENCES}

[AS] M. Atiyah, I. M. Singer, The Index of Elliptic Operators on Compact Manifolds, Bull. Amer. Math. Soc. 69 (1963), 422-433. MR 26:626

[BW] B. Booß-Bavnbek, K. Wojciechowski, Elliptic Boundary Problems for Dirac Operators, Birkhäuser, Boston Basel Berlin, 1993. MR 94h:58168

[CM] A. Connes, H. Moscovici, The $L^{2}$-Index Theorem for Homogeneous Spaces of Lie Groups, Ann. Math. 115 (1982), 291-330. MR 84f:58108

[G] S. Goldberg, Unbounded Linear Operators, Theory and Applications, Dover Publications, Inc., New York, 1985. MR 86k:47001

[GL] M. Gromov, H. B. Lawson, Jr., Positive Scalar Curvature and the Dirac Operator on Complete Riemannian Manifolds, Publ. Math. IHES 58 (1983), 295-408. MR 85g:58082

[L] A. Lichnerowicz, Spineurs Harmoniques, C. R. Acad. Sci. Paris, Ser. A-B 257 (1963), 7-9. MR 27:6218

[LM] H. B. Lawson Jr., M.-L. Michelsohn, Spin Geometry, Princeton Univ. Press, Princeton, NJ, 1989. MR 91g:53001

[RS] M. Reed, B. Simon, Methods of Modern Mathematical Physics. Vol. II: Fourier Analysis, Self-Adjointness, Academic Press, New York San Francisco London, 1975. MR 58:12429b

[S] I. M. Singer, Families of Dirac Operators with Applications in Physics, Élie Cartan et les Mathématiques d'Aujourd'hui, Astérisque, Soc. Math. France, Paris, 1985, pp. 323340. MR 88a:58192

Department of Mathematics, University of North Texas, Denton, Texas 76203

E-mail address: anghel@sol.acs.unt.edu 